1985

\title{
Photoinduced nucleation of carbon disulfide
}

Ofra Kalisky

Richard H. Heist

Fairfield University, rheist@fairfield.edu

Follow this and additional works at: https://digitalcommons.fairfield.edu/engineering-facultypubs Copyright 1985 American Institute of Physics

The final publisher PDF has been archived here with permission from the copyright holder. https://aip.scitation.org/doi/abs/10.1063/1.449122

\section{Peer Reviewed}

\section{Repository Citation}

Kalisky, Ofra and Heist, Richard H., "Photoinduced nucleation of carbon disulfide" (1985). Engineering Faculty Publications. 153.

https://digitalcommons.fairfield.edu/engineering-facultypubs/153

\section{Published Citation}

Kalisky, O., \& Heist, R. H. (1985). Photoinduced nucleation of carbon disulfide. The Journal of chemical physics, 83(7), 3668-3680. doi:10.1063/1.449122.

This item has been accepted for inclusion in DigitalCommons@Fairfield by an authorized administrator of DigitalCommons@Fairfield. It is brought to you by DigitalCommons@Fairfield with permission from the rightsholder(s) and is protected by copyright and/or related rights. You are free to use this item in any way that is permitted by the copyright and related rights legislation that applies to your use. For other uses, you need to obtain permission from the rights-holder(s) directly, unless additional rights are indicated by a Creative Commons license in the record and/or on the work itself. For more information, please contact digitalcommons@fairfield.edu. 


\title{
Photoinduced nucleation of carbon disulfide
}

\author{
Ofra Kalisky and Richard $\mathrm{H}$. Heist ${ }^{\mathrm{a})}$ \\ Department of Chemical Engineering, University of Rochester, Rochester, New York 14627
}

(Received 7 October 1983; accepted 18 June 1985)

\begin{abstract}
The action of light on undersaturated and supersaturated vapors of carbon disulfide has been investigated using a batch photochemical reactor and a thermal diffusion cloud chamber, respectively. Photoinduced nucleation was observed in each case. In the batch reactor enough sulfur was produced to nucleate and grow a sulfur aerosol. A model for the photoinduced nucleation of supersaturated carbon disulfide is proposed based upon the photochemical production and subsequent nucleation of sulfur. The model predictions compare well with observed nucleation delay time and nucleation rate data. A variation of the model utilizing diradical polymerization instead of nucleation is used to explain photoinduced nucleation results in the literature involving dilute solutions of carbon disulfide in supersaturated ethanol vapor.
\end{abstract}

\section{INTRODUCTION}

The action of light on supersaturated vapors has been of interest for many years. In the last decade, this interest has increased sharply and has given rise to a significant increase in the number of related publications. ${ }^{1-11}$ The majority of the work utilizes the thermal diffusion cloud chamber (TDCC) to produce and maintain the supersaturated host vapor, and much of the interest has focused upon the utility of the TDCC (the supersaturated vapor, actually) to detect very low levels of contaminants. For instance, the light induced disproportionation reaction involving $\mathrm{SO}_{2}$ in supersaturated water vapor has been studied in which $\mathrm{SO}_{3}$ and, eventually $\mathrm{H}_{2} \mathrm{SO}_{4}$, are produced. ${ }^{1}$ Binary nucleation of $\mathrm{H}_{2} \mathrm{SO}_{4}$ and water is observed for which the concentration of $\mathrm{H}_{2} \mathrm{SO}_{4}$ has been estimated to be as small as 1000-10000 molecules/cc.

Although photoinduced nucleation (PIN) has been observed in a variety of systems, there are only a few for which the intermediate(s) responsible for the nucleation has been identified. In much of the literature describing PIN experiments, the substance responsible for the initial light absorption is identified, and the material involved in the final condensation and growth process is known (the host supersaturated vapor); but the identity of intermediate species remains a mystery. Long-lived, host molecule stabilized "photoaffected" species, ${ }^{2-5}$ free radicals, ${ }^{6-10}$ and low vapor pressure reaction products $1,10,11$ are commonly postulated to account for observed PIN behavior, and a phenomenological model is formulated to further evaluate the PIN data. The extreme sensitivity of the supersaturated vapor and the low concentration of the intermediate make further identification difficult. The purpose of this paper is to identify the intermediate involved in the PIN of carbon disulfide.

PIN involving carbon disulfide in supersaturated ethanol has been reported in the literature ${ }^{2}$ In that investigation, a long-lived, host molecule stabilized "photoaffected" species was postulated to account for the observed PIN behavior. Although these authors used low concentrations of carbon disulfide and, thus, ruled out sulfur as the cause of the PIN, the complex chemistry of sulfur and the multiplicity of reaction paths involving sulfur and carbon disulfide

\footnotetext{
a) To whom all correspondence should be addressed.
}

make it difficult to ignore sulfur playing a role in the PIN. Our approach has been to accept the possibility of sulfur as an intermediate and, instead of postulating host molecule stabilized clusters, consider other mechanisms, e.g., sulfur nucleation and diradical polymerization, as possible explanations for the PIN in carbon disulfide.

There are two parts to the experimental investigation described in this paper: batch experiments involving irradiation of carbon disulfide and carbon disulfide-helium mix. tures in a photochemical cell designed to identify the product of the action of light on carbon disulfide and PIN experiments using supersaturated carbon disulfide in a TDCC. Results of the TDCC experiments are analyzed in terms of a phenomenological model which incorporates the results of the cell experiments. The last part of the investigation involves a comparison of our model predictions with experimental data from the carbon disulfide-ethanol system taken from the literature.

\section{EXPERIMENTAL}

The design and operation of the TDCC used in this investigation is similar to that described elsewhere ${ }^{12}$ so a detailed explanation need not be repeated here. There are, however, a few significant differences that deserve comment. The cloud chamber plates used in this investigation have been constructed from titanium-stabilized steel and coated with a $0.025 \mathrm{~cm}$ thick layer of a refractory glass ${ }^{13}$ specially formulated to be highly resistant to chemical attack. ${ }^{14}$ The ring used to separate the chamber plates and form the chamber interior is fused silica chosen to provide high transmittance ( $>80 \%$ ) at wavelengths down to $200 \mathrm{~nm}$. Finally, teflon gaskets were used to separate the fused silica ring and the chamber plates. ${ }^{15}$ The chamber interior contains only glass, fused silica, and teflon.

The gas handling apparatus used to control the environment inside the TDCC was constructed of Pyrex, teflon, and Viton (there are Viton O rings in two Cajon Ultratorr fittings used to connect the TDCC to the vacuum line and Viton 0 rings in the stopcocks used in the vacuum line). The vacuum line and the helium carrier gas supply were trapped with either liquid nitrogen or a dry ice-methanol slush. Results were the same in either case. The temperature and partial 
TABLE I. Expressions for carbon disulfide equilibrium vapor pressure $\boldsymbol{P}_{e}$, molar heat capacity $c_{p}$, thermal conductivity $\lambda$, binary diffusion coefficient $D_{a b}$, surface tension $\sigma$, vapor viscosity $\eta$, liquid density $\rho$, and molecular weight. ${ }^{2}$ Expressions for carrier gas properties are available in Ref. 12.

$$
\begin{array}{ll}
\hline \hline \text { Carbon disulfide: } & \\
P_{e}=P_{0}+\sum_{i=1}^{7} P_{i} T^{i} & P_{0}=153.67 \\
& P_{1}=-3.3299 \\
& P_{2}=0.01818 \\
& P_{3}=-2.9399 \times 10^{-6} \\
& P_{4}=-5.3927 \times 10^{-8} \\
& P_{5}=-4.3259 \times 10^{-10} \\
& P_{6}=-1.5650 \times 10^{-12} \\
c_{p}=c_{0}+\sum_{i=1}^{3} c_{i} T^{i} & P_{7}=1.0542 \times 10^{-14} \\
& C_{0}=6.555 \\
& C_{1}=1.941 \times 10^{-2} \\
\lambda=\lambda_{0}+\sum_{i=1}^{3} \lambda_{i} T^{i} & C_{2}=-1.831 \times 10^{-5} \\
& C_{3}=6.384 \times 10^{-9} \\
& \lambda_{0}=-2.6678 \times 10^{-6} \\
& \lambda_{1}=4.4671 \times 10^{-8} \\
& \lambda_{2}=1.1379 \times 10^{-10} \\
& \lambda_{3}=-1.1881 \times 10^{-13}
\end{array}
$$

$D_{a b}^{0}(273 \mathrm{~K}, 1200 \mathrm{~mm} \mathrm{Hg})^{e}=0.2559 ; D_{a b}=D_{a b}^{0}\left(\frac{T}{273}\right)^{1.72}\left(\frac{1200}{P_{T}}\right)^{i}$

$=35.28-0.1472(T-273)^{f}$

$\eta=\frac{1.1 \times 10^{-5} T^{1.58}}{(T+283)}$

$\rho=\rho_{0}+\sum_{i=1}^{3} \rho_{i} T^{h}$

$$
\begin{aligned}
& \rho_{0}=1.8940 \\
& \rho_{1}=-3.673 \times 10^{-3} \\
& \rho_{2}=8.12 \times 10^{-6} \\
& \rho_{3}=-1.94 \times 10^{-8}
\end{aligned}
$$

$\mathrm{MW}=76.131$

' $\lambda$ in cal $/(\mathrm{cm} \mathrm{s} \mathrm{K}), P_{e}$ in $\mathrm{mm} \mathrm{Hg}, \sigma$ in erg $/ \mathrm{cm}^{2}, \eta$ in poise, $\rho$ in $\mathrm{g} / \mathrm{cm}^{3}, c_{p}$ in $\mathrm{cal} /(\mathrm{mol} \mathrm{K}), D_{a b}$ in $\mathrm{cm}^{2} / \mathrm{s}, T$ in $\mathrm{K}$, and $P_{T}$ in $\mathrm{mm} \mathrm{Hg}$.

'The polynomial is a 7th order regression for data from the literature over the temperature range of interest: (i) G. Waddington et al., J. Phys. Chem. 66, 1074 (1962); T. Boublik and A. Aim, Collect. Czech. Chem. Commun. 37, 3513 (1972); D. N. Seshadri et al., J. Indian Inst. Sci. A 50, 295 (1968). 'R. C. Reid, J. M. Prausnitz, and T. K. Sherwood, The Properties of Gases and Liquids (McGraw-Hill, New York, 1977), p. 634.

'Footnote c, p. 478, Eq. (10-3.11) was used to estimate vapor thermal conductivity; calculated data fit to 3 rd order polynomial.

'Footnote c, pp. 549, 678; R. H. Perry, Chemical Engineering Handbook, Sth ed. (McGraw-Hill, New York, 1973), pp. 3-231-3-234.

'Int. Crit. Tables 4, 447 (1928).

'R. H. Perry, Chemical Engineering Handbook, 5th ed. (McGraw-Hill, New York, 1973), p. 3-211.

Int. Crit. Tables 3, 23 (1928).

Estimated using the method of E. N. Fuller, P. D. Schettler, and J. C. Giddings, I\&EC 58, 19 (1966).

pressure profiles in the TDCC were calculated using methods described elsewhere. ${ }^{12,16}$ The physical property data used for these calculations are listed in Table I.

The batch photochemical reactor experiments were carried out using a fused silica cell, $2.5 \mathrm{~cm}$ in diameter and 10 $\mathrm{cm}$ long. The cell was attached to the same gas handling system described above.

Both the TDCC and the fused silica cell were cleaned prior to every set of new experiments. The cleaning method involved washing with hot, chromium free cleaning acid (NOCHROMIX), multiple rinsing with doubly distilled water and, in some cases, final rinsing with the working fluid to be used in the experiment. A detergent wash was also used on occasion, but results were the same. Reagent grade carbon disulfide (NCB 99.9\%), absolute ethanol (99\%), and helium (Airco, 99.99\%) were used in this investigation without further purification. The distilled water was prepared by first distilling from an alkaline permanganate solution and then distilling a second time.

For the TDCC experiments, a Molectron $U V-12$ pulsed nitrogen laser or a Schoeffel $1000 \mathrm{~W}$ high pressure xenon arc lamp was used as an excitation source. A Bausch and Lomb $0.25 \mathrm{~m}$ monochromator was used with the xenon arc lamp for wavelength selection. A combination of slits and fused silica lenses were used to shape the excitation beam into a rectangular geometry $(0.2 \mathrm{~cm}$ thick and $4.2 \mathrm{~cm}$ wide) and direct it through the TDCC at the maximum of the supersaturation profile (about $75 \%$ of the chamber height). For the fused silica cell experiments, the nitrogen laser beam was used and collimated or focused in the center of the cell with fused silica lenses. In both the cell and the TDCC, particles formed by photoinduced nucleation were detected as they passed through a helium-neon laser beam and scattered light to a photomultiplier tube. The scattering events in the TDCC were recorded using an electronic counter interfaced to a microcomputer. ${ }^{17}$ In the cell experiments, the scattered laser light was detected using standard phase sensitive methods and displayed on a chart recording. Light intensity measurements were made using a volume absorbing disc calorimeter (Scientech, Model 38-0105). Variations in incident light intensity were made using combinations of Corning filters or by varying the repetition rate of the nitrogen laser. The absorbance of all filter combinations were determined prior to the investigation using a GCA McPherson spectrophotometer. The repetition rate of the nitrogen laser was varied from 0 to 8 pulses/s. The average power was determined to be directly proportional to the repetition rate over this range.

\section{RESULTS}

The first part of this section presents data obtained from the batch experiments using the fused silica photochemical cell. The second part presents data obtained from PIN experiments on supersaturated carbon disulfide in the TDCC.

\section{A. Cell experiments}

The objective of the photochemical cell experiments was to identify the material produced by the action of light on carbon disulfide.

Carbon disulfide and mixtures of carbon disulfide and helium at partial pressures comparable to those used in TDCC experiments were irradiated in the cell with a pulsed nitrogen laser. During a typical experiment, both the nitrogen laser and the helium-neon laser beams were directed through the same region of the cell. After an initial delay time,${ }^{18}$ an aerosol was observed to form in the excitation beam and then gradually spread throughout the cell. After 
extended irradiation, a yellow deposit was observed along the bottom of the cell directly below the path of the excitation beam. Aerosol formation was observed over a wide range $(\sim 10-300 \mathrm{~mm} \mathrm{Hg})$ of carbon disulfide pressures. Eventually, for carbon disulfide pressures below about 10 $\mathrm{mm} \mathrm{Hg}$, the time for aerosol formation became sufficiently long that we no longer observed an aerosol during the course of the experiment. The deposits from these experiments were analyzed using mass spectroscopy and optical absorption spectroscopy and were found to be sulfur. The only other materials found in the deposit were a trace amount of carbon monosulfide and residual carbon disulfide. It is important to note two points: In all cases the sulfur aerosol formed only in the excitation beam, it did not form near the cell walls and the carbon disulfide vapor was undersaturated. The aerosol was due entirely to sulfur condensation and growth not to carbon disulfide condensation. There was enough sulfur produced by the action of light on carbon disulfide to nucleate and grow sulfur particles of greater than micron size.

\section{B. Sulfur chemistry}

Although we have qualitatively identified the primary product of the action of light on carbon disulfide, we cannot yet be certain of its quantitative nature because of the complexities of the chemistry and photochemistry of sulfur. ${ }^{19-24}$ For instance, sulfur shows a marked tendency to form multiple S-S bonds, resulting in a wide variety of polymeric allotropic forms: eight membered rings, larger and smaller rings, and linear chains ranging in length from 2 to over 10000 atoms. ${ }^{24-27}$ Most of these species, however, have only limited stability. The high sensitivity of the S-S bonds to light and to the conditions of formation and storage increase the complexity of sulfur chemistry.
In the solid, the orthorhombic allotrope $\left(\mathbf{S}_{8}\right)$ is the most stable crystalline form to which all the other metastable forms transform (usually quite slowly) at room temperature. ${ }^{20,24}$ The yellow crystals consist of "crown" or "chair" shaped eight-membered rings. ${ }^{20}$ This is consistent with the results of our mass spectral analysis in which the $S_{8}$ peak was dominant. In the liquid, brown polysulfides have been observed to form, irreversibly, in the presence of trace organic impurities (even in sulfur which is $99.999 \%$ pure). ${ }^{26}$ This may explain the origin of a light brown tint to the yellow deposit we observed on a few occasions during our cell experiments although, we never observed mass spectra peaks which could be attributed to organic impurities. In the liquid, it is well known that thermal sission of $\mathrm{S}_{8}$ rings (at elevated temperatures) gives rise to the formation of linear sulfenyl diradicals which, in turn, lead to extensive polymerization. ${ }^{24-31}$ In fact, due to very fast propagation steps, sulfur polymers (in the liquid) typically have 50000 or more sulfur atoms.

At lower temperatures saturated sulfur vapor consists primarily of the $S_{8}$ species. ${ }^{26,32,33}$ At elevated temperatures or lower pressures, however, the vapor is a mixture of the $S$, $S_{2}, S_{4}, S_{6}$, and $S_{8}$ species. Apparently, as the pressure is lowered, the lower molecular weight allotropes become increasingly more common.

Sulfur polymers can easily be produced at room temperature by photolytic dissociation. ${ }^{20,24,34-37}$ The sensitivity of the S-S bond to light (even sunlight) has been demonstrated. ${ }^{26}$ In both solution and the solid, the cyclooctosulfur molecule is scissioned by light and found to undergo a complex series of propagation and transfer reactions yielding sulfur polymers, regenerated $S_{8}$ molecules, as well as other species. ${ }^{34}$ The sensitivity of the $\mathrm{S}-\mathrm{S}$ bond to light is also demonstrated by the polysulfides. ${ }^{38-42}$ Many of them undergo vinyl
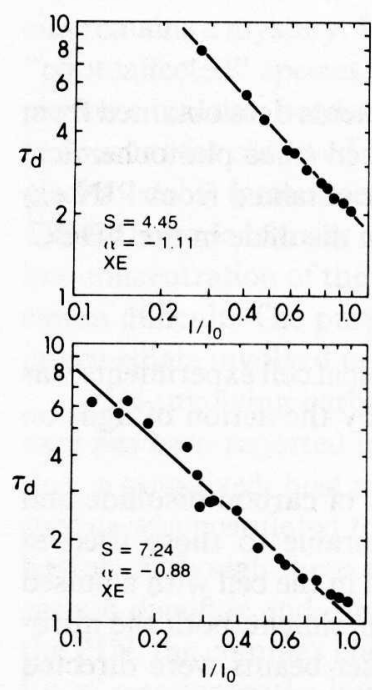
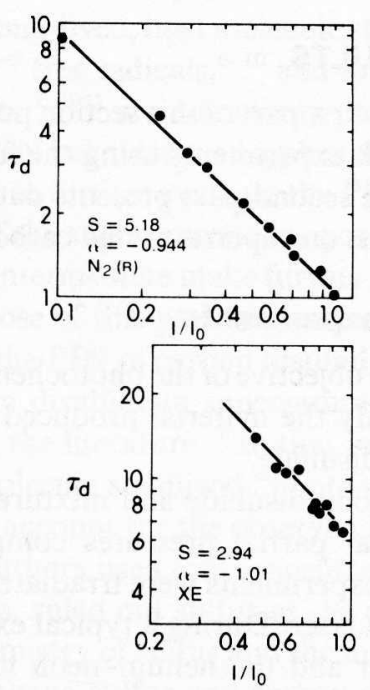

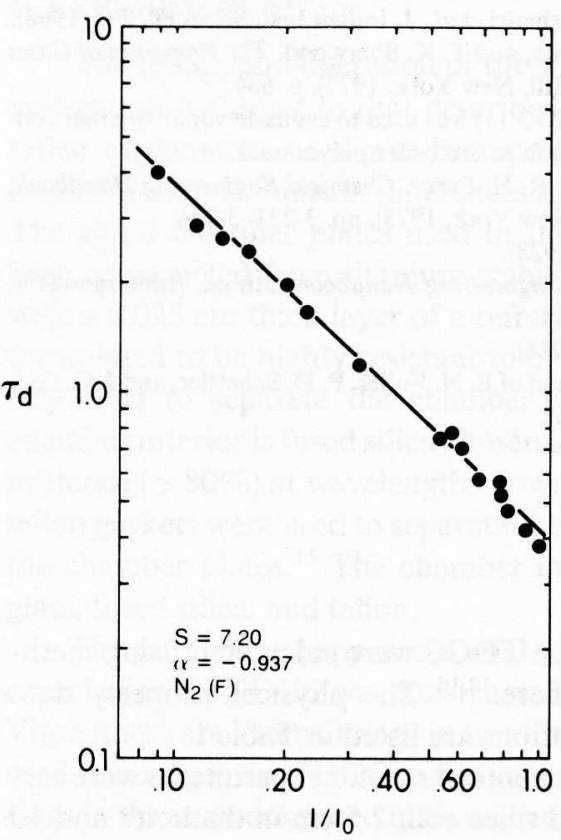

FIG. 1. Representative plots of delay time $t_{d}$ vs light intensity $I / I_{0}$ for PIN in supersaturated carbon disulfide. The notation on each plot indicates the carbon disulfide supersaturation $S$, the slope of the plot $\alpha$, and the type of excitation, respectively. Xe indicates the xenon arc lamp and $\mathrm{N}_{2}$ the nitrogen laser. $(f)$ and $(r)$ indicate that the light intensity variation was accomplished with optical filters or by varying the laser repetition rate, respectively. See the text for details. 
polymerization at room temperature, even polysulfides which do not tend to undergo thermal polymerization.

\section{TDCC experiments}

There is little doubt that during our experiments light absorption by carbon disulfide is the primary photophysical event or that the observed nucleation is photoinduced. When there is no source of optical excitation, there is no nucleation. With carbon disulfide in the chamber, optical excitation gives rise to nucleation only as long as the wavelength of light corresponds to the optical absorption of carbon disulfide. In fact, stepping through the first absorption band of carbon dilsulfide with the xenon lamp-monochromator assembly gives rise to an excitation spectrum (number of droplets as a function of wavelength) in agreement with the absorption band of carbon disulfide. This is consistent with results obtained by other investigators. ${ }^{2}$ The PIN results are the same whether or not an external electric field $(135 \mathrm{~V} / \mathrm{cm})$ is applied across the cloud chamber plates indicating there is no photoionization occurring.

Two kinds of experiments are described in this section: nucleation delay time measurements and photoinduced nucleation rate measurements. The nucleation delay time is the time from the beginning of irradiation to the onset of nucleation. Each set of delay time measurements were made for a range of incident light intensities at a fixed carbon disulfide supersaturation, and each set of data corresponds to a different carbon disulfide supersaturation (all at approximately $250 \mathrm{~K})$. Plotting the logarithm of the delay time vs the logarithm of the light intensity (in the form of $I / I_{0}$, the fraction of light incident on the TDCC) for a particular supersaturation gives rise to a straight line for each set of data. For convenience, only representative plots of these data are shown inFig. 1, however, the slopes of all of the curves are given in Table II. Within our experimental uncertainty, the slopes of all of the curves are the same, independent of carbon disulfide supersaturation. The average value for all of the slopes is $-1.03 \pm 0.14$

The nucleation rate experiments are steady state measurements in which the carbon disulfide was irradiated and the steady nucleation count rate measured as a function of light intensity at a particular supersaturation (all at approximately $250 \mathrm{~K}$ ). Nucleation rate (drops/cc/s) is directly proportional to nucleation count rate (drops $/ 10 \mathrm{~s}$ ). For generality, the analysis in this paper is presented in terms of nucleation rate; the data, however, are reported as nucleation count rate. At times these two terms are referred to interchangeably, but this should cause no confusion. The results are, of course, unaffected by this definition. The nucleation rates were kept small to avoid vapor depletion and latent heat of condensation effects. ${ }^{16,43,44}$ When the logarithm of the nucleation count rate is plotted vs the logarithm of the light intensity $\left(I / I_{0}\right)$ at a particular supersaturation, a straight line is obtained. For convenience, only representative plots of these data are shown in Fig. 2, however, the slopes of all of the curves at different supersaturations are given in Table II. Within experimental uncertainty, the slopes of all the lines are the same, independent of the carbon disulfide supersaturation. The average value for these slopes
TABLE II. Delay time and photoinduced nucleation count rate data for carbon disulfide. Part A is a list of the slopes of the $\log \left(t_{d}\right)$ vs $\log (I)$ plots at constant supersaturation. The mode of excitation is indicated. The designation $(f)$ or $(r)$ indicates intensity variation using optical filters or varying the laser repetition rate, respectively. Part B is a list of the slopes of the $\log (R)$ vs $\log (I)$ plots at constant supersaturation. Part $C$ is a list of the slopes of the $\log (R)$ vs $\log \left(\mathrm{CS}_{2}\right)$ plots at constant light intensity. As in the text, $I / I_{0}$ is abbreviated simply as $I$. The xenon arc lamp was used for parts $B$ and $C$. See the text for details.

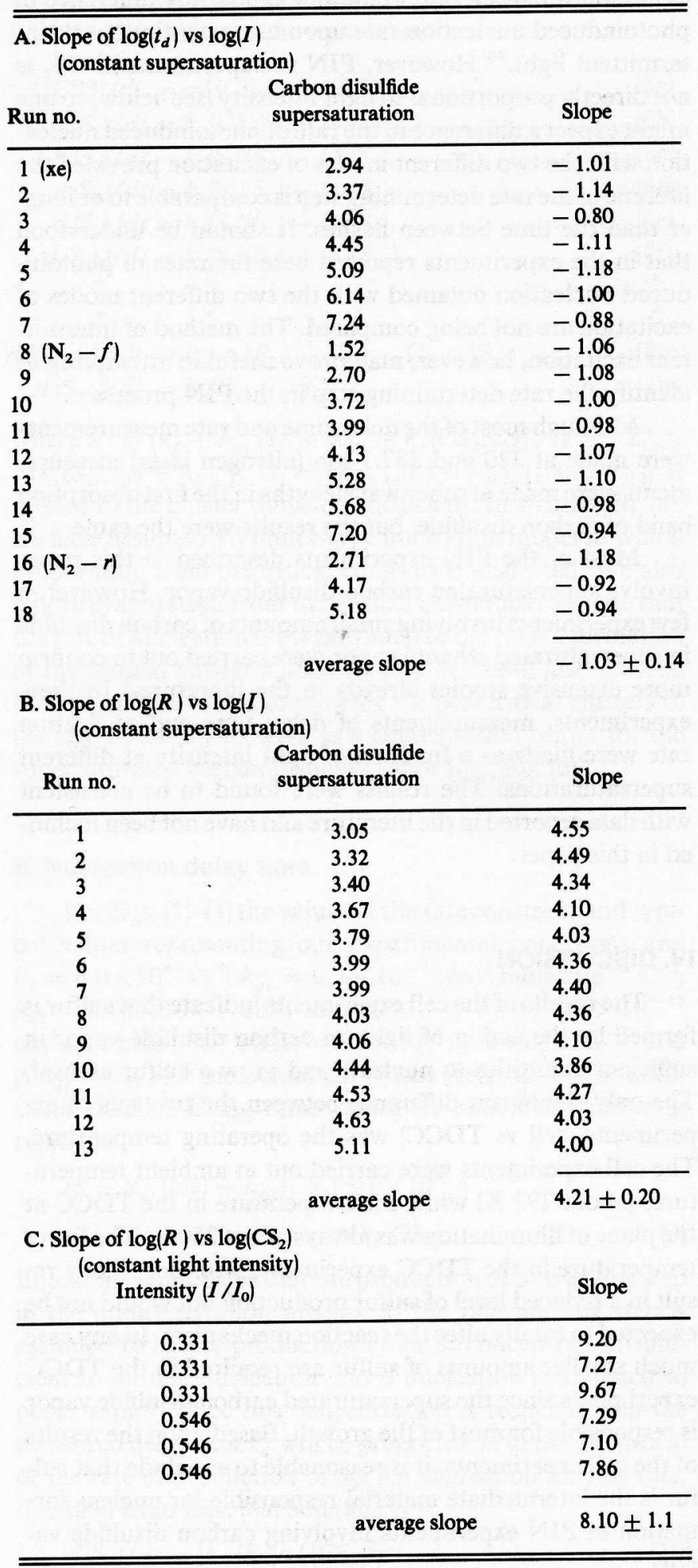

is $4.21 \pm 0.2$. This behavior, observed for both the delay time and the nucleation rate, differs from results obtained from similar experiments on carbon disulfide in ethanol ${ }^{2,45}$ and from results obtained in other PIN investigations. ${ }^{3-5,10}$ 
Both the nucleation delay time and rate measurements were made using the xenon arc lamp-monochromator assembly (continuous excitation) as well as the nitrogen laser (pulsed excitation). Since the slopes of both the delay time and the nucleation rate vs light intensity plots do not appear to depend upon the excitation source, there is no effect due to intermittent excitation. If the PIN were directly proportional to light intensity, one would not expect any difference in photoinduced nucleation rate upon using continuous or intermittent light. ${ }^{46}$ However, PIN in supersaturated $\mathrm{CS}_{2}$ is not directly proportional to light intensity (see below) so one might expect a difference in the rate of photoinduced nucleation with the two different modes of excitation provided the lifetime of the rate determining step is comparable to or longer than the time between flashes. It should be understood that in the experiments reported here the rates of photoinduced nucleation obtained with the two different modes of excitation are not being compared. The method of intermittent excitation, however, may prove useful in attempting to identify the rate determining step in the PIN process.

Although most of the delay time and rate measurements were made at 320 and $337.1 \mathrm{~nm}$ (nitrogen laser) measurements were made at other wavelengths in the first absorption band of carbon disulfide, but the results were the same.

Most of the PIN experiments described in this paper involve supersaturated carbon disulfide vapor. However, a few experiments involving small amounts of carbon disulfide in supersaturated ethanol vapor were carried out to confirm more extensive studies already in the literature. ${ }^{2}$ In these experiments, measurements of delay time and nucleation rate were made as a function of light intensity at different supersaturations. The results were found to be consistent with data reported in the literature and have not been included in this paper.

\section{DISCUSSION}

The results of the cell experiments indicate that sulfur is formed by the action of light on carbon disulfide vapor in sufficient quantities to nucleate and grow a sulfur aerosol. The only significant difference between the two sets of experiments (cell vs TDCC) was the operating temperature. The cell experiments were carried out at ambient temperatures (about $293 \mathrm{~K}$ ) while the temperature in the TDCC at the plane of illumination was always about $250 \mathrm{~K}$. The lower temperature in the TDCC experiments will most likely result in a reduced level of sulfur production but would not be expected to totally alter the reaction mechanism. In any case much smaller amounts of sulfur are required in the TDCC experiments since the supersaturated carbon disulfide vapor is responsible for most of the growth. Based upon the results of the cell experiments, it is reasonable to conclude that sulfur is the intermediate material responsible for nucleus formation in PIN experiments involving carbon disulfide vapor.

The fact that the slopes of the delay time-light intensity and nucleation rate-light intensity curves are independent of supersaturation is significant. It implies that sulfur clusters are nucleated and then grow by sulfur addition until they are large enough to serve as a condensation center for the supersaturated carbon disulfide.

This picture, however, is at odds with data from experiments involving low partial pressures of carbon disulfide in supersaturated ethanol vapor. ${ }^{2,45}$ In these experiments the slopes of both the delay time and nucleation rate data depend upon ethanol supersaturation. Apparently, at the low concentrations of carbon disulfide used in these experiments (of the order of $3000 \mathrm{ppm}$ and less) the amount of sulfur produced was insufficient to nucleate and grow sulfur clusters. Instead, another mechanism which results in a significant ethanol supersaturation dependence must be responsible for the cluster growth. We shall return to this point later, but it is important to recognize that there are (at least) two regimés of carbon disulfide partial pressure to consider: high pressure (of the order of 100 Torr) and low pressure (of the order of 1 Torr and less).

\section{A. High pressure regime}

We do not know the quantitative chemical nature of the sulfur intermediate(s) in the PIN process. There are a number of possibilities; but, based upon the results of the cell experiments and the sulfur chemistry discussion presented earlier, it is probable that $S_{2}$ is involved. See the Appendix for a discussion of an alternative mechanism. If we arbitrarily limit our consideration to $S_{2}$, we can write the following general mechanism for $\mathrm{CS}_{2}$ vapor PIN:

$\begin{array}{rlll}\mathrm{CS}_{2}+h v & \rightarrow \mathrm{CS}_{2}^{*}, & \sigma I & (1) \\ \mathrm{CS}_{2}^{*} & \rightarrow \mathrm{CS}_{2}+h v^{\prime}, & k_{f} & (2) \\ \mathrm{CS}_{2}^{*}+\mathrm{He} & \rightarrow \mathrm{CS}_{2}+\mathrm{He}, & k_{q 1} & (3 \mathrm{a}) \\ \mathrm{CS}_{2}^{*}+\mathrm{CS}_{2} & \rightarrow 2 \mathrm{CS}_{2}, & k_{q 2} & (3 \mathrm{~b}) \\ \mathrm{CS}_{2}^{*}+\mathrm{CS}_{2} & \rightarrow 2 \mathrm{CS}+\mathrm{S}_{2}, & k_{r} & (4) \\ \mathrm{S}_{2}+\mathrm{S}_{2} & \rightarrow \mathrm{S}_{4}, & k_{2} & (5 \mathrm{a}) \\ \mathrm{S}_{4}+\mathrm{S}_{2} & \rightarrow \mathrm{S}_{6}, & k_{3} & (5 \mathrm{~b}) \\ \mathrm{S}_{6}+\mathrm{S}_{2} & \rightarrow \mathrm{S}_{8}, & k_{4} & (5 \mathrm{c}) \\ \mathbf{S}_{8}+\mathrm{S}_{2} & \rightarrow \mathrm{S}_{10}, & k_{5} & (5 \mathrm{~d}) \\ " \prime \prime & " & k_{6} & (5 \mathrm{e}) \\ " \prime & & k_{7} & (5 \mathrm{f}) \\ \mathrm{S}_{2(n-1)}+\mathrm{S}_{2} & \rightarrow \mathrm{S}_{2 n}, & k_{n} & (5 \mathrm{n}) \\ \mathrm{S}_{2 n}+p \mathrm{~S}_{2} & \rightarrow \mathrm{S}_{2(n+p)}, & & (6) \\ S_{2(n+p)}+\mathrm{qCS}_{2} & \rightarrow \text { droplet. } & & (7)\end{array}$

It must be emphasized once again that while we are confident sulfur is being produced, we are assuming, for the purpose of providing a mechanistic description, that $S_{2}$ is directly involved in the actual condensation process. Our objective is to demonstrate that PIN in CS $_{2}$ vapor can be explained using a reasonable model involving sulfur production. In fact, we show in the Appendix that our experimental data can also be explained using other sulfur allotropes in place of $S_{2}$ in the above mechanism.

In this mechanism, Eq. (1) represents the initial carbon disulfide photoexcitation. The absorption cross section is $\sigma$ and the incident light intensity I. Equations (2) and (3) represent alternative quenching pathways for the phtoexcited carbon disulfide. Equation (4) is the production of sulfur and the byproduct carbon monosulfide from the photoexcited carbon disulfide. Equation (5) represents the nucleation of $S_{2}$ to form a critical nucleus. Equation (6) represents the growth of 


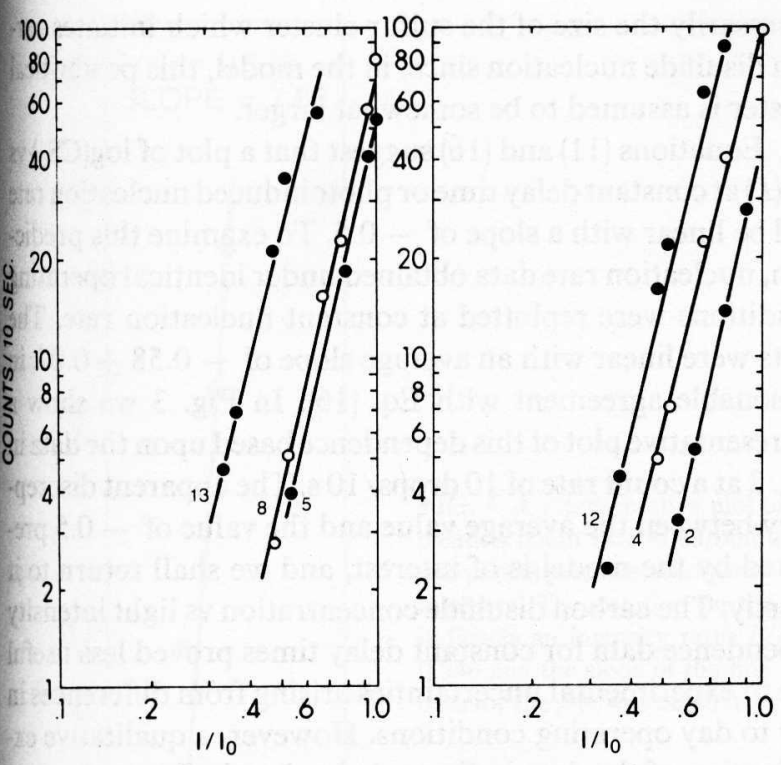

FIG. 2. Representative plots of photoinduced nucleation count rate vs light intensity. The numbers on each plot refer to the Run no.'s in Table II. The count rate is directly proportional to the nucleation rate $R$ used in the text.

the post critical cluster to a size sufficient to serve as a condensation center for the supersaturated carbon disulfide vapor. Equation (7) represents the final carbon disulfide growth process producing a droplet which is detected in the TDCC.

It is necessary at this point to comment upon the fate of the carbon monosulfide shown being produced in Eq. (4). There is convincing evidence that both $\mathrm{S}_{2}$ molecules and CS radicals are produced when $\mathrm{CS}_{2}$ vapor is irradiated with light in the wavelength range $280-360 \mathrm{~nm}^{47-50}$ The lifetime of the sulfur produced is short (e.g., $300-400 \mu \mathrm{s}$ ) and is believed to be due to rapid formation of higher sulfur homologs. ${ }^{47}$ The lifetime of the CS produced is much longer (e.g., from several minutes to a few seconds depending upon the cell history) and is believed to be due to heterogeneous deactivation of the CS at the cell wall. ${ }^{47,48}$ In our cell experiments, a yellow (or slightly brownish-yellow deposit, depending upon the total $\mathrm{CS}_{2}$ pressure) collected along the bottom of the cell directly below the path of the excitation beam. A much less obvious brownish colored deposit could also be observed uniformly coating the interior of the cell during the course of the longer experiments. These observations suggest that nucleated sulfur was responsible for the aerosol which formed and settled to the bottom of the cell, and that CS diffusing to the wall and deactivated there was responsible for the uniform brownish coating. This explanation accounts for the small amount of CS found in the mass spectra ( $\mathrm{CS}_{2}$ cracking and residual CS deposited on the aerosol particles before reaching the wall). Because of the small sample size available, only the deposit on the bottom of the cell was analyzed with the mass spectrograph. There is evidence that the deactivation of CS at the cell walls produces a carbon-rich deposit which acts to further enhance the deactivation process producing more carbon-rich material and regenerating $\mathrm{CS}_{2}{ }^{48}$ We heated one side of a sample cell with a torch and observed a silvery-gray filamentlike condensate form on the cooler side of the cell. Since certain allotropic forms of carbon are of this texture and color, ${ }^{51}$ this observation is, at least, consistent with the formation of carbon-rich material at the cell wall during the deactivation of CS. There is also evidence that the amount of sulfur formed by the action light on $\mathrm{CS}_{2}$ has no effect upon the lifetime of $\mathrm{CS}^{47}$ This also suggests that the primary deactivation of CS is at the cell wall.

The differential equations governing the evolution of Eqs. (1)-(5) in time can be written as:

$$
\begin{aligned}
& d\left(\mathrm{CS}_{2}^{*}\right) / d t=\sigma I\left(\mathrm{CS}_{2}\right)-\mathrm{k}^{\prime}\left(\mathrm{CS}_{2}^{*}\right), \\
& d\left(\mathrm{~S}_{2}\right) / d t=k_{r}\left(\mathrm{CS}_{2}\right)\left(\mathrm{CS}_{2}^{*}\right)-\Omega\left(\mathrm{S}_{2}\right) \text {, } \\
& d\left(\mathbf{S}_{4}\right) / d t=k_{2}\left(\mathbf{S}_{2}\right)\left(\mathbf{S}_{2}\right)-\Omega_{2}\left(\mathbf{S}_{4}\right) \text {, } \\
& d\left(\mathbf{S}_{6}\right) / d t=k_{3}\left(\mathbf{S}_{4}\right)\left(\mathbf{S}_{2}\right)-\Omega_{3}\left(\mathbf{S}_{6}\right) \text {, } \\
& d\left(\mathbf{S}_{8}\right) / d t=k_{4}\left(\mathbf{S}_{6}\right)\left(\mathbf{S}_{2}\right)-\Omega_{4}\left(\mathbf{S}_{8}\right) \text {, } \\
& d\left(\mathbf{S}_{10}\right) / d t=k_{5}\left(\mathbf{S}_{8}\right)\left(\mathbf{S}_{2}\right)-\Omega_{5}\left(\mathbf{S}_{10}\right) \text {, } \\
& \text { " " } \\
& d\left(\mathbf{S}_{2 n-2}\right) / d t=k_{n-1}\left(S_{2 n-4}\right)\left(\mathbf{S}_{2}\right)-\Omega_{n-1}\left(\mathbf{S}_{2 n-2}\right), \\
& d\left(\mathrm{~S}_{2 n}\right) / d t=k_{n}\left(\mathrm{~S}_{2 n-2}\left(S_{2}\right)\right. \text {, }
\end{aligned}
$$

where $k^{\prime}=\left(k_{f}+k_{q 1} \mathrm{He}+\left(k_{q 2}+k_{r}\right) \mathrm{CS}_{2}\right) \mathrm{CS}_{2}^{*} ; \Omega=\left[\Sigma_{i=2}^{n}\right.$ $\left.\times k_{i}\left(S_{2 i-2}\right)+k_{D 2}\right] ; \Omega_{j}=\left[k_{j+1}\left(\mathrm{~S}_{2}\right)+k_{D 2 j}\right]$, and $k_{D 2 j}$ is related to the cluster diffusion coefficient. In writing Eq. (8), we have assumed a Volmer-type nucleation model in which evaporation from precritical embryos is neglected. We also ignore evaporation from the critical cluster and assume that the rate of nucleation is proportional to the rate of formation of the critical cluster $k_{n}\left(\mathbf{S}_{2 n-2}\right)\left(\mathbf{S}_{2}\right)$. We have also ignored the loss of $S_{2}$ involved in growing the postcritical clusters to the size necessary to serve as a condensation center for the supersaturated carbon disulfide vapor [i.e., Eq. (6)].

\section{B. Nucleation delay time}

For Eqs. (1)-(4) the values of the rate constants and typical values representing our experimental conditions are: $k_{f}=6.0 \times 10^{4} \mathrm{~s}^{-1} ; k_{q 1}=0.2 \times 10^{-10} \mathrm{~cm}^{3}$ molecule $^{-1} \mathrm{~s}^{-1}$; $k_{q 2}=0.76 \times 10^{-10} \mathrm{~cm}^{3}$ molecule ${ }^{-1} \mathrm{~s}^{-1} ; k_{r}=6 \times 10^{-15}$ $\mathrm{cm}^{3}$ molecule ${ }^{-1} \mathrm{~s}^{-1} ;(\mathrm{He})=40 \times 10^{18}$ molecules $\mathrm{cm}^{-3}$; and $\left(\mathrm{CS}_{2}\right)=6 \times 10^{18}$ molecules $\mathrm{cm}^{-3}$ (see Refs. $52-58$ for additional details). Using these values we obtain the following relations:

$$
\begin{aligned}
k_{f}+k_{q 1}(\mathrm{He})+k_{q 2}\left(\mathrm{CS}_{2}\right) & \simeq 1 \times 10^{9} \mathrm{~s}^{-1} \\
& >k_{r}\left(\mathrm{CS}_{2}\right) .
\end{aligned}
$$

Equation (9) indicates that the production of $S_{2}$ is a slow step in the photoexcitation process. If the rate limiting step is assumed to be the production of an $\mathbf{S}_{2}$ concentration sufficient to initiate nucleation and if nucleation is assumed to occur rapidly once this concentration is reached, then the measured delay time $t_{d}$ will be proportional to the reciprocal of the rate of production of $S_{2}$. An expression for this rate, obtained from Eqs. (8a) and (8b), is

$$
d\left(\mathrm{~S}_{2}\right) / d t=\left(k_{r} / k^{\prime}\right) \sigma I\left(\mathrm{CS}_{2}\right)^{2},
$$

where we have used the stationary state approximation to obtain an expression for $\mathrm{CS}_{2}^{*}$ and neglected $\mathrm{S}_{2}$ losses arising from the second term in Eq. (8b) (i.e., short time behavior). If we equate the delay time to the reciprocal of Eq. (10) (using a 
suitable constant of proportionality) and express the resulting equation in logarithmic form, we have

$$
\log \left(t_{d}\right)=-\log (I)-2 \log \left(\mathrm{CS}_{2}\right)+\log \left(\mathrm{H}_{2}\right) .
$$

In Eq. (11), $\mathrm{H}_{2}$ is a (nearly) constant term which depends weakly on carbon disulfide concentration. For the present discussion, $\mathrm{H}_{2}$ will be assumed constant, but we shall return to this point later. In writing Eq. (11), we have abbreviated $I$ / $I_{0}$ simply as $I$, for convenience. We shall follow this convention from this point on. Equation (11) suggests that a plot of the logarithm of the delay time against the logarithm of the light intensity will have a slope of -1 , and that this slope will be independent of the carbon disulfide concentration. Both of these predictions are in good agreement with the measured slopes listed in Table II.

\section{Photoinduced nucleation rate}

During steady photoinduced nucleation, the right-hand sides of Eq. (8) vanish, and the behavior of the model is given by the solution of the resulting algebraic equations describing the distribution of precritical clusters obtained from Eqs. $(8 \mathrm{c})$ to $(8 \mathrm{~m})$. As described above, the rate of photoinduced nucleation, $R$, is assumed to be proportional to the rate of production of critical clusters $\left[k_{n}\left(\mathrm{~S}_{2 n-2}\right)\left(\mathrm{S}_{2}\right)\right]$ so solving the resulting algebraic equations for $\left(\mathbf{S}_{2 n-2}\right), R$ can be expressed as:

$$
R=K_{2}^{\prime}\left(\mathrm{S}_{2}\right)^{n} .
$$

In deriving Eq. (12), we have assumed a steady $S_{2}$ concentration and that cluster diffusion is the important loss mechanism. In Eq. (12),

$$
K_{2}^{\prime}=\frac{k_{n} k_{n-1} \cdots k_{2}\left(V_{c}\right)}{k_{D 2 n-2} k_{D 2 n-4} \cdots k_{D 4}} B,
$$

where $V_{c}$ is the counting volume (the actual volume in the TDCC giving rise to the drops being counted for the nucleation rate measurement); $B$ is the proportionality term relating nucleation rate to the rate of critical cluster formation and the other terms have already been defined. The $S_{2}$ concentration in Eq. (12) can be expressed in terms of experimental observables by obtaining the steady solution to Eqs. (8a) and (8b). Substituting the expression for this solution into Eq. (12) gives:

$$
R=K_{2}(I)^{n}\left(\mathrm{CS}_{2}\right)^{2 n},
$$

where

$$
K_{2}=k_{r} \sigma K_{2}^{\prime} /\left(k^{\prime} k_{D 2}\right) .
$$

In determining $S_{2}$ we have again assumed diffusion to be the important loss mechanism. Expressing Eq. (14) in logarithmic form gives:

$$
\log (R)=n \log (I)+2 n \log \left(\mathrm{CS}_{2}\right)+\log \left(K_{2}\right) .
$$

$K_{2}$ is a (nearly) constant term depending weakly on carbon disulfide concentration. For now $K_{2}$ will be assumed constant, but we shall return to this later. Equation (16) suggests that a plot of $\log (R)$ vs $\log (I)$ will be linear with slope $n$, the number of $S_{2}$ molecules in the critical size cluster. The data in Fig. 2 and Table II are consistent with the form of Eq. (16). The average value of 4.21 obtained for the slope implies that the critical cluster contains 4 or $5 \mathrm{~S}_{2}$ molecules. This is not necessarily the size of the sulfur cluster which initiates carbon disulfide nucleation since, in the model, this postcritical cluster is assumed to be somewhat larger.

Equations (11) and (16) suggest that a plot of $\log \left(\mathrm{CS}_{2}\right)$ vs $\log (I)$ at constant delay time or photoinduced nucleation rate will be linear with a slope of -0.5 . To examine this prediction, nucleation rate data obtained under identical operating conditions were replotted at constant nucleation rate. The plots were linear with an average slope of $-0.58 \pm 0.07$, in reasonable agreement with Eq. (16). In Fig. 3 we show a representative plot of this dependence based upon the data in Fig. 2 at a count rate of 10 drops $/ 10 \mathrm{~s}$. The apparent discrepancy between the average value and the value of -0.5 predicted by the model is of interest, and we shall return to it shortly. The carbon disulfide concentration vs light intensity dependence data for constant delay times proved less useful due to experimental uncertainties arising from differences in day to day operating conditions. However, a qualitative examination of the data indicates behavior similar to the constant nucleation rate data with slopes ranging from -0.4 to -0.6 , in approximate agreement with Eq. (11).

As a further test of the model, Eq. (16) suggests that a plot of $\log \left(\mathrm{CS}_{2}\right)$ vs $\log (R)$ at constant $I$ should be linear with a positive slope twice that of the curves in Fig. 2. To examine this prediction, nucleation rate data obtained under similar operating conditions were plotted at constant $I / I_{0}$. The plots were linear with an average slope of $8.1 \pm 1.1$, again, in reasonable agreement with Eq. (16). The individual values for the slopes are listed in Table II. In Fig. 4 we show a representative plot of this dependence prepared from the data shown in Fig. 2 at $I / I_{0}=0.546$.

The apparent discrepancy referred to earlier can be traced to the expression for the steady concentration of $\mathrm{CS}_{2}^{*}$ in the reaction model. The complete expression for the rate of change of $\mathrm{CS}_{2}^{*}$ is

$$
d \mathrm{CS}_{2}^{*} / d t=\sigma I\left(\mathrm{CS}_{2}\right)-\mathbf{k}^{\prime}\left(\mathrm{CS}_{2}^{*}\right),
$$

where $k^{\prime}$ has already been defined. If $k^{\prime}$ is assumed constant, Eqs. (11) and (16) follow. If $k^{\prime}$ is allowed to depend upon carbon disulfide concentration, Eqs. (11) and (16) assume the following forms.

$$
\log \left(t_{d}\right)=-\log (I)-2 \log \left(\mathrm{CS}_{2}\right)+\log \left(k^{\prime}\right)-\log \left(L_{2}\right)(18)
$$
and

$$
\log (R)=n \log I+2 n \log \left(\mathrm{CS}_{2}\right)-n \log \left(k^{\prime}\right)-\log \left(J_{2}\right),
$$

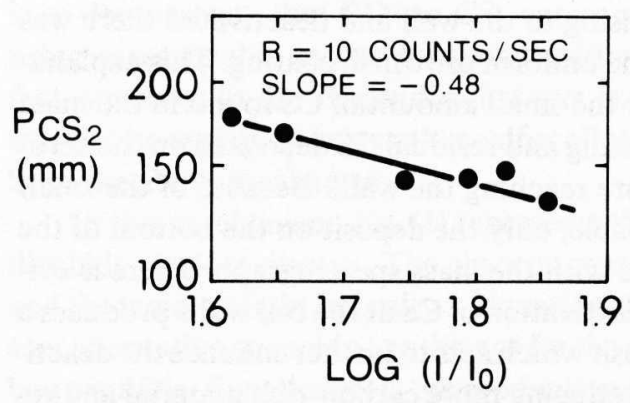

FIG. 3. A representative plot of carbon disulfide partial pressure $P_{\mathrm{Cs}_{2}}$ vs light intensity at constant count rate. The notation on the plot indicates a count rate of 10 drops $/ 10 \mathrm{~s}$ and the slope of the line. The data in the plot was taken from Fig. 2. 


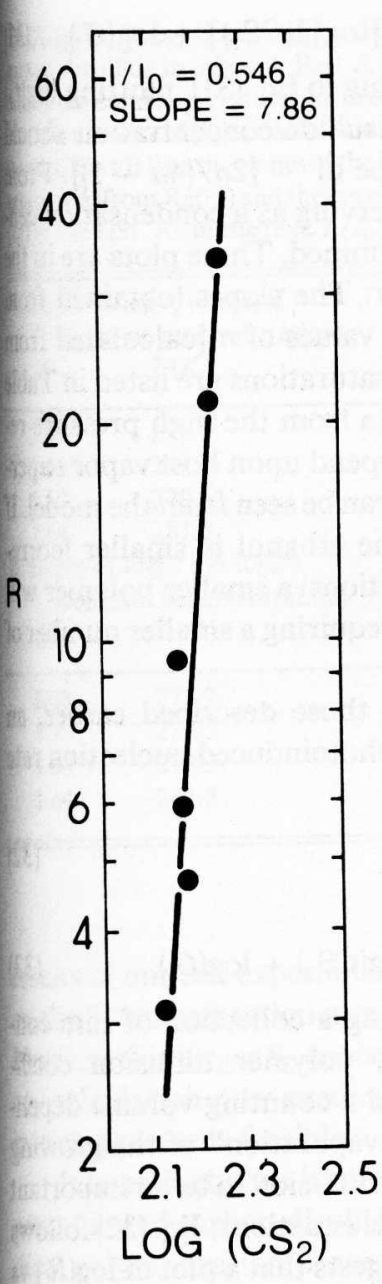

where $L_{2}$ and $K_{2}$ are constants and the other terms have already been defined. The additional terms in Eqs. (18) and (19) will not effect the $\log (R)$ vs $\log (I)$ dependence or the $\log \left(t_{d}\right)$ vs $\log (I)$ dependence but will effect the $\log \left(\mathrm{CS}_{2}\right)$ vs $\log (I)$ dependence at constant nucleation rate or constant delay time because of the $\mathrm{CS}_{2}$ dependence in $k^{\prime}$. Examining Eqs. (18) and (19) more closely reveals that including the additional term will change the slope predicted by the model to larger negative values in agreement with the data from the nucleation rate experiments. A similar argument applies to the $\log \left(t_{d}\right)$ vs $\log \left(\mathrm{CS}_{2}\right)$ dependence at constant intensity. This also accounts for the observation that the slope of the curve in Fig. 4 is less than twice that of the curves in Fig. 2.

We have used Eq. (19) in place of Eq. (16) and have found slightly improved agreement with the predicted slope. It can be seen that $k^{\prime}$ does not depend strongly upon $\mathrm{CS}_{2}$ if the terms in $k^{\prime}$ are examined more closely. If values for the individual rate constants in $k^{\prime}$ and conditions typical of our experiments are considered, the value of $k_{f}+k_{q l}(\mathrm{He})$ is found to be 3 to 4 times larger than the $\left(k_{q 2}+k_{r}\right)\left(\mathrm{CS}_{2}\right)$ term. This would tend to make $k^{\prime}$ only moderately sensitive to variations in $\mathrm{CS}_{2}$. For this reason, we have used Eqs. (11) and (16) and suppressed the carbon disulfide dependence in $k^{\prime}$.

\section{Low pressure regime}

We have already stated that the PIN data reported in this paper (high pressure regimé) do not agree with PIN data in the literature from experiments involving small concen- trations of carbon disulfide in supersaturated ethanol (low pressure regimé). This implies that either sulfur is not involved in that PIN process (as suggested in the literature ${ }^{2}$ ) or that the sulfur nucleation mechanism proposed in Eqs. (5)(6) is not applicable in the low pressure regime.

The rate of production of $S_{2}$ in the low pressure regimé has been calculated, based on literature values for $k_{r}$ [the reaction rate constant for $S_{2}$ production in Eq. (4) above] and has been shown to be too small to produce sufficient $S_{2}$ to permit nucleation. ${ }^{2}$ However, if the literature value for $k_{r}$ is used for comparison with the results of our cell experiments, a serious discrepancy emerges. If we estimate the approximate size and particle density of the sulfur aerosol formed in the photochemical cell after a certain irradiation period, we can compare this with predictions for sulfur production based on the value of $k_{r}$ from the literature. In fact, using typical values for aerosol number density and size, ${ }^{58}$ the calculated time to form the aerosol is about three orders of magnitude longer than actually observed. Although our estimation of number density was certainly qualitative, it was most likely low since we only counted the particles in the excitation beam (thereby missing all of the particles having moved out of the beam either by convective motion, gravitational settling, or diffusion) and we could only see the particles that grew large enough to scatter a significant amount of light from the excitation beam.

The possibility that $k_{r}$ may be larger than previously thought is significant. It means previous conclusions based upon the literature value of $k_{r}$ are questionable. An increase of 1000 in the value of $k_{r}$ will significantly increase the amount of $S_{2}$ in the model PIN calculations and, although it will still not be enough to initiate nucleation, ${ }^{2}$ it is enough to support alternative mechanisms of sulfur particle production.

Remaining consistent with our approach in the investigation, we continue to accept the possibility of sulfur participating in the PIN process. Rather than assume the existence of a host molecule stabilized "photoaffected" species, we assume (consistent with the results of our cell experiments) sulfur to be responsible for the PIN in the low pressure regime. What remains is to develop a model consistent with the cell results, the sulfur chemistry literature, and the experimental PIN results already in the literature for the carbon disulfide/ethanol system.

It has already been noted that $S_{2}$ is increasingly common in sulfur vapors at lower pressures. It is also noted that, since the light intensity in the TDCC is higher in the low pressure regime (ethanol is transparent and the carbon disulfide concentration is small) and since sulfur bonds are particularly sensitive to light, most of the sulfur produced will very likely be continually re-excited. This is an important assumption since, as was pointed out in the Sulfur Chemistry Section, optical excitation of sulfur commonly leads to diradical formation.

If we assume sulfur is in the form of $S_{2}$ and allow for widespread diradical formation, the following mechanism can be written

$$
\begin{array}{rlrl}
\mathrm{CS}_{2}+h v & \rightarrow \mathrm{CS}_{2}^{*}, & \sigma I \\
\mathrm{CS}_{2}^{*} & \rightarrow \mathrm{CS}_{2}+h v^{\prime}, & & k_{f}
\end{array}
$$




$$
\begin{aligned}
& \mathrm{CS}_{2}^{*}+\mathrm{He} \rightarrow \mathrm{CS}_{2}+\mathrm{He} \text {, } \\
& \mathrm{CS}_{2}^{*}+\mathrm{Eh} \rightarrow \mathrm{CS}_{2}+\mathrm{Eh} \text {, } \\
& \mathrm{CS}_{2}^{*}+\mathrm{CS}_{2} \rightarrow 2 \mathrm{CS}+\mathrm{S}_{2} \text {, } \\
& \mathrm{S}_{2}+h v^{\prime} \rightarrow \mathrm{S}_{2}^{*} \text {, } \\
& \mathrm{S}_{2}^{*} \rightarrow \mathrm{S}_{2}+h v^{\prime} \text {, } \\
& \mathbf{S}_{2}^{*}+\mathbf{A} \rightarrow \mathbf{S}_{2}+\mathbf{A} \text {, } \\
& S_{2}^{*} \rightarrow\left(\cdot S_{2} \cdot\right) \\
& \left(\cdot S_{2} \cdot\right)+\left(\cdot S_{2^{*}}\right) \rightarrow\left(\cdot S_{2^{\prime}} \cdot\right)_{2} \text {, } \\
& \left(\cdot \mathrm{S}_{2^{\circ}}\right)_{2}+\left(\cdot \mathrm{S}_{\left.\mathbf{2}^{\circ}\right)}\right) \rightarrow\left(\cdot \mathrm{S}_{2}\right)_{3} \text {, } \\
& \left(\cdot S_{2} \cdot\right)_{3}+\left(\cdot S_{2} \cdot\right) \rightarrow\left(\cdot S_{2} \cdot\right)_{4} \text {, } \\
& \left(\cdot S_{2} \cdot\right)_{n-1}+\left(\cdot S_{2} \cdot\right) \rightarrow\left(\cdot S_{2} \cdot\right)_{n}, \\
& \left(\cdot \mathrm{S}_{2} \cdot\right)_{n}+q \mathrm{Eh} \rightarrow \text { droplet. }
\end{aligned}
$$

In Eq. (20), $\sigma$ is the absorption coefficient for carbon disulfide. Helium $(\mathrm{He})$ and ethanol $(\mathrm{Eh})$ are represented as quenching agents in Eqs. (22a) and (22b). We have ignored self-quenching of the $\mathrm{CS}_{2}$. Equation (23) represents the formation of sulfur and carbon monosulfide. ${ }^{47,48}$ The first five steps are similar to those in the high pressure regime except that ethanol has replaced carbon disulfide as a quenching agent. In Eq. (24a), $\sigma^{\prime}$ is the absorption coefficient for $\mathrm{S}_{2}$. Equation (24c) represents a quenching process where $A$ can be ethanol or helium. In Eq. (24d) the sulfur diradical is produced. In Eqs. (25a) to (25n) the sulfur undergoes free radical polymerization until the sulfur polymer becomes large enough to serve as a condensation center for the supersaturated ethanol. At this point Eq. (26) the ethanol condenses eventually producing a droplet which is detected during the experiment. We ignore the presence of the carbon monosulfide for the same reasons given earlier in the discussion following Eqs. (1)-(7).

We first consider the short time behavior of the model. If we ignore diffusive losses and polymer "evaporation", and if we assume the photoinduced nucleation rate is proportional to the concentration of the $\left(\cdot S_{2} \cdot\right)_{n-1}$ species indicated in Eq. (25n), the following expression for the photoinduced nucleation rate can be written:

$$
R \propto \lambda_{n}\left(\cdot \mathrm{S}_{2}\right)\left(\cdot \mathrm{S}_{2} \cdot\right)_{n-1} .
$$

If $\left(\cdot S_{2} \cdot\right)_{n-1}$ is evaluated using the model equations, Eq. (27) becomes

$$
R=G^{\prime \prime}\left\{\left[\mathrm{I}\left(\mathrm{CS}_{2}\right)\right]^{2}\right\}^{n[4(n-1)+2]},
$$

where $G^{\prime \prime}$ is a collection of rate constants, absorption coefficients, numerical constants, and a term related to the counting volume in the TDCC and is taken to be constant. In arriving at Eq. (28), we have also assumed that during diradical production [Eqs. (24a) to (24d)] Eq. (24a) is rate limiting and that Eq. (24d) is the predominant decay mechanism. To obtain the delay time, Eq. (28) is integrated and set equal to unity:

$$
1=\int_{0}^{t_{d}} R d t^{\prime}
$$

and

$$
1=\left[G^{\prime}\left[\mathrm{I}\left(\mathrm{CS}_{2}\right)\right]^{2 n}\right] t_{d}^{(4 n-1)} .
$$

Solving Eq. (30) for the delay time and rewritting in terms of logarithms gives:

$$
\log \left(t_{d}\right)=-[2 n /(4 n-1)] \log \left[\mathrm{I}\left(\mathrm{CS}_{2}\right)\right]+\log (G),
$$

where $G$ is a constant. According to Eq. (31), plotting $\log \left(t_{d}\right)$ vs $\log (I)$ a constant carbon disulfide concentration should give a straight line with a slope of $-[2 n /(4 n-1)]$. From this the size $n$ of the polymer serving as a condensation center for the ethanol can be determined. These plots are in the literature, ${ }^{2}$ and they are linear. The slopes (obtained from Ref. 2) and the corresponding values of $n$ [calculated from Eq. (31)] for a number of supersaturations are listed in Table III. In contrast to the PIN data from the high pressure regime, the slopes in Table III depend upon host vapor supersaturation. The reason for this can be seen from the model. If the critical nucleus size for the ethanol is smaller (corresponding to higher supersaturations) a smaller polymer will serve as a condensation center requiring a smaller number of "excitations".

Using methods similar to those described earlier, an expression for the steady state photoinduced nucleation rate can be written as

$$
R=O\left(\mathrm{CS}_{2}\right)^{2 n} \mathrm{I}^{2 n}
$$

or, in terms of logarithms,

$$
\log (R)=2 n \log (I)+2 n \log \left(\mathrm{CS}_{2}\right)+\log (O),
$$

where $O$ is a constant containing a collection of rate constants, adsorption coefficients, polymer diffusion coefficients, numerical constants, and a counting volume dependent term. We have ignored "evaporation" of the growing polymers and assumed polymer diffusion to be an important loss mechanism. Using the model equations, Eq. (32) follows from Eq. (27). Equation (33) suggests that a plot of $\log (R)$ vs $\log (I)$ at constant supersaturation will be linear with a slope of $2 n$. These plots are available in the literature, ${ }^{2}$ and they are linear. The slopes (obtained from Ref. 2) and the calculated value of $n$ [from Eq. (33)] are listed in Table III. The measured slopes are observed to depend upon ethanol supersaturation which, as with the delay time, is consistent with the model. The values of $n$ obtained from the delay time data and the nucleation rate data at the same supersaturations are, to within experimental uncertainty, identical.

Equations (31) and (33) predict that a plot of $\log \left(\mathrm{CS}_{2}\right)$ vs $\log (I)$ at constant delay time or nucleation rate, respectively, will give a slope of -1 . This dependence has been observed ${ }^{2}$ and the slope found to be -1 , in agreement with the model.

The model for PIN in the low pressure regime does not require a sulfur concentration large enough for nucleation. It does require a sufficiently large number of sulfenyl diradicals to allow the free radical polymerization to proceed at observable rates. We can estimate an upper bound for the sulfenyl concentration by assuming all the $S_{2}$ formed is continually re-excited to the diradical form and then examine the $S_{2}$ concentration using simple kinetic theory arguments to see if the polymerization required by the model is, indeed, feasible.

The steady $\mathrm{S}_{2}$ concentration in these experiments has been estimated to be about $5.0 \times 10^{3}$ molecules/cc, not enough for nucleation. ${ }^{2}$ Simple collision theory predicts that this concentration is also not sufficient for the free radical polymerization process suggested by our model to occur. However, if a value for $k_{r}$ is used which is consistent with the 
TABLE III. Delay time and photoinduced nucleation count rate data for arbon disulfide in ethanol. Part A lists the slopes of the $\log \left(t_{d}\right)$ vs $\log (I)$ dats at constant supersaturation (from Ref. 2) and the corresponding value of $h$, the number of $\left(S_{2}\right)$ molecules in the polymer, obtained from Eq. (26) in thetext. Part B lists the slopes of the $\log (R)$ vs $\log (I)$ data at constant supersturation (from Ref. 2) and the corresponding value of $n$ obtained from Eq. (28) in the text. As in the text, $I / I_{0}$ is abbreviated simply as $I$.

\begin{tabular}{|c|c|c|c|}
\hline \multicolumn{4}{|c|}{$\begin{array}{l}\text { A Slopes of } \log \left(t_{d}\right) \text { vs } \log (I) \\
\text { (constant supersaturation) }\end{array}$} \\
\hline$S$ & $T(K)$ & Slope & $n$ \\
\hline 2.08 & 275.9 & $-0.542 \pm 0.006$ & 3.23 \\
\hline & 277.5 & $-0.536 \pm 0.004$ & 3.72 \\
\hline 1.80 & 279.3 & $-0.525 \pm 0.007$ & 5.25 \\
\hline 1.64 & 281.5 & $-0.517 \pm 0.004$ & 7.60 \\
\hline \multicolumn{4}{|c|}{$\begin{array}{l}\text { B. Slopes of } \log (R) \text { vs } \log (I) \\
\text { (constant supersaturation) }\end{array}$} \\
\hline$S$ & & Slope & $n$ \\
\hline 2.08 & 275.9 & $6.25 \pm 0.35$ & 3.13 \\
\hline 1.95 & 277.5 & $7.82 \pm 0.24$ & 3.91 \\
\hline 1.80 & 279.3 & $11.16 \pm 0.54$ & 5.58 \\
\hline 1.64 & 281.5 & $15.85 \pm 1.14$ & 7.93 \\
\hline
\end{tabular}

results of our cell experiments, i.e., roughly 1000 times larg$e r$, the $\mathrm{S}_{2}$ concentration increases by a factor of at least 1000 . If we use this larger $\mathrm{S}_{2}$ concentration, the same collision theory calculation now predicts that the free radical polymerization process is feasible.

Interestingly, we have been able to describe PIN in supersaturated carbon disulfide without including the free radical polymerization process just discussed. While it seems reasonable that diradical formation must also occur in the high pressure regime, it apparently does not play a significant role. It may be that sulfur acts as a chain terminator and suppresses the polymerization process, whereas in the low pressure regime the sulfur concentration is sufficiently small that this process is of lesser importance. Evidence from the literature supports this since sulfur in solution is known to be an efficient radical trap and sulfenyl radicals, like sulfide ions, are exceptionally thiophilic. ${ }^{59}$

In this paper we have presented an alternative explanation for PIN in carbon disulfide based upon the photochemical formation of sulfur. Unfortunately, sulfur chemistry is sufficiently complicated that the simple model we propose is unlikely to be definitive. For instance, in our treatement we have ignored the existence of impurities. It is known that even trace amounts of water (less than a fraction of a percent) promote reaction with carbon disulfide in the presence of light (even sunlight) producing formic acid, atomic sulfur, and hydrogen sulfide. ${ }^{60}$ Hydrogen sulfide reacts with ethanol to produce mercaptans which are known to produce free radicals when irradiated with ultraviolet light. ${ }^{61}$ Further complicating the picture is the reaction of carbon disulfide with ethanol to produce alkane thiols. ${ }^{62}$ This process normally requires a catalyst, but in the presence of light it may occur. Thiols are known to produce free radicals when irradiated with ultraviolet light. ${ }^{61}$ In addition, the presence of organic material in the TDCC (e.g., gaskets) allows the possibility of organic vapor contamination. With this contamination, free radical formation or low vapor pres- sure photochemical reaction products are possible both of which can lead to nucleation.

The role of impurities in PIN has been impressively demonstrated by experiments with the water-helium system in the TDCC. Details of the experiments will not be given here, ${ }^{10}$ but the salient features are of interest since they illustrate the importance of trace contaminates.

In these experiments, scrupulously cleaned water ${ }^{63}$ was used as a working fluid with helium as a carrier gas. The chamber was irradiated with ultraviolet light and PIN was observed only for wavelengths less than approximately 240 $\mathrm{nm}$. Measurements of the light intensity dependence of the photoinduced nucleation rate at constant supersaturation similar to those described above were made. The $\log (R)$ vs $\log (I)$ plots were observed to be linear with slopes dependent upon water vapor supersaturation, quite similar to the carbon disulfide in ethanol results described above. The slopes from the water vapor experiments are shown in Fig. 5 as a function of the water vapor supersaturation to illustrate the dependences observed. The critical nucleus size for the supersaturated water vapor is also plotted in Fig. 5 for the range of supersaturation. Interestingly, the dependence of the critical nucleus size on supersaturation appears identical to that of the slopes of the rate vs intensity data. This suggests multiple "excitations" were required to produce the condensation nucleus, similar to the model for the carbon disulfide low pressure regime described above. It should be mentioned here that a plot constructed in a manner identical to Fig. 5 for the carbon disulfide-ethanol experiments exhibits the same type of behavior as shown in Fig. 5.

Of related interest is a description from the literature ${ }^{11}$ in which similar PIN experiments involving water vapor and helium were attempted with the important difference that the cloud chamber (now an expansion chamber) was constructed entirely of quartz. All organic materials, e.g., gaskets, were excluded from the chamber. Under these conditions no PIN was observed. These results strongly suggest the role of trace contaminates in many PIN processes.

\section{SUMMARY}

Photoinduced nucleation of carbon disulfide vapor has been investigated for both supersaturated carbon disulfide and small amounts of carbon disulfide in supersaturated ethanol. Spectroscopic analysis of the results of batch reactor studies involving ultraviolet irradiation of carbon disulfide vapor indicate that sulfur is the dominant product of the action of light on carbon disulfide. Assuming sulfur to be the intermediate in the PIN process and using the known photochemistry of carbon disulfide and sulfur, two mechanisms have been proposed to explain PIN in systems containing carbon disulfide. The first, for supersaturated carbon disulfide, involves sulfur production and nucleation and growth of sulfur clusters followed by condensation of carbon disulfide. The mechanism predicts the dependence of the nucleation delay time and the steady state photoinduced nucleation rate on light intensity to be independent of carbon disulfide supersaturation, which is confirmed by experiment. It predicts that the dependence of the logarithm of carbon disulfide supersaturation on the logarithm of light intensity nec- 


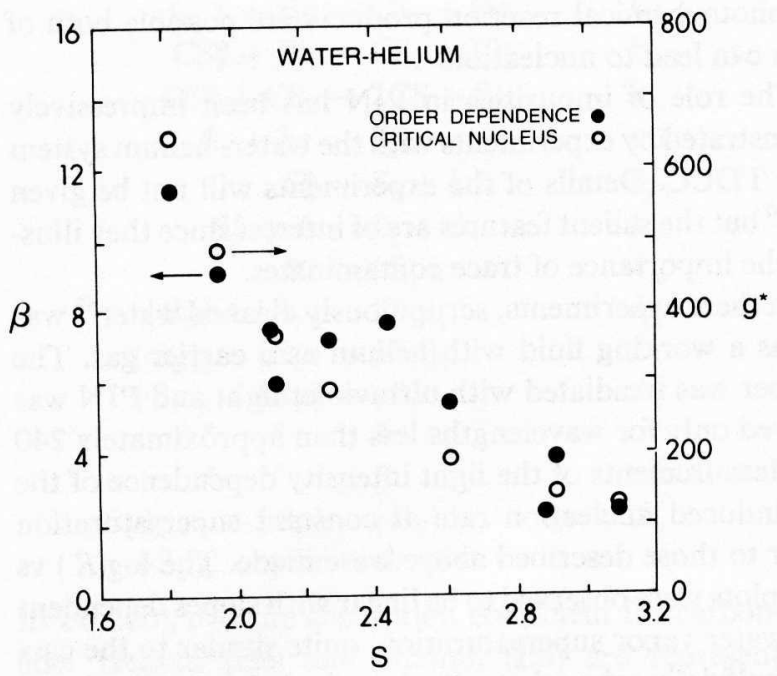

FIG. 5. The variation of the order $\beta$ of the light intensity vs the supersaturation for the water vapor PIN experiments described in the text. The variation of the critical nucleus size for water vs supersaturation is also shown. See Ref. 10 for details.

essary to maintain a constant delay time or a constant nucleation rate should be approximately -0.5 , which is also confirmed by experiment. The mechanism also predicts that the slope of the $\log \left(\mathrm{CS}_{2}\right)$ vs $\log (R)$ data at constant light intensity will be twice that of the $\log (R)$ vs $\log (I)$ data at constant carbon disulfide concentration. This is confirmed by experiment.

The second mechanism, for small amounts of carbon disulfide in supersaturated host vapors, involves sulfur production and subsequent excitation forming diradicals which undergo free radical polymerization to a size sufficient to nucleate the host vapor. The mechanism predicts the dependence of the delay time and the nucleation rate on light intensity to be a function of the host supersaturation, which is confirmed by experiment both in the author's laboratory and by results from the literature. It also predicts that the dependence of the logarithm of the carbon disulfide concentration on the logarithm of the light intensity necessary to maintain a constant delay time or a constant nucleation rate should be -1 , which is confirmed by results from the literature.

By assuming the identity of the PIN intermediate to be sulfur, PIN in carbon disulfide vapor can be explained. It is not necessary to invoke long-lived "photoaffected" species to explain the nucleation. It may be that the sulfur diradicals do, indeed, interact with the host molecules. This could help explain the small values of $n$ in Table III and also why such (apparently) small amounts of sulfur are so effective in nucleating a supersaturated vapor. The free radicals are reactive, and there are other examples of a nucleation barrier being lowered by interaction with host molecules, e.g., ioninduced nucleation.

One other comment should be made concerning the use of the TDCC in identifying the intermediates in the PIN process. Both delay time and decay time (time from cutting off the excitation beam until no further nucleation is observed) are conveniently made with the TDCC assembly described above, and since diffusion is the primary loss mechanism for the products of the PIN process, it should be possible to use the delay and decay time data to obtain diffusion coefficient information about the intermediates. This information may be useful in identifying PIN intermediates.

\section{ACKNOWLEDGMENT}

The authors gratefully acknowledge the assistance of Mr. Yeong Choi in carrying out the mass spectral analysis of the sulfur deposits in the photochemical cell experiments.

\section{APPENDIX}

Because of the variety of sulfur allotropes, it is possible to formulate other mechanisms which are consistent with the results of the PIN experiments. For instance, consider $\mathrm{S}_{8}$. This allotrope is the most stable form of sulfur and one might expect the reactive $S_{2}$ to quickly form the $S_{8}$ species which then would nucleate and grow eventually serving as a condensation center for the supersaturated carbon disulfide. The most serious objection to this mechanism arises from the amount of sulfur necessary to support the process. For example, if we assume experimental conditions typical of the PIN experiments in the high pressure regime and a value of $k_{r}$ consistent with the results of the cell experiments (see Ref. 58 ) the rate of $S_{2}$ production is about $2.0 \times 10^{10}$ molecules/ $\mathrm{cc} / \mathrm{s}$ and the steady $\mathrm{S}_{2}$ concentration is approximately $10^{11}$ molecules/cc. Simple collision theory calculations suggest that $S_{8}$ formation is possible, but subsequent nucleation of $S_{8}$ becomes increasingly difficult. If, for the purposes of this discussion, we allow the possibility of $\mathrm{S}_{8}$ formation and nucleation, then a mechanism for this process (in the high pressure regimé) is

$$
\begin{array}{cl}
\mathrm{CS}_{2}+h v \rightarrow \mathrm{CS}_{2}^{*}, & \sigma I \\
\mathrm{CS}_{2}^{*} \rightarrow \mathrm{CS}_{2}+h v^{\prime}, & k_{f} \\
\mathrm{CS}_{2}^{*}+\mathrm{He} \rightarrow \mathrm{CS}_{2}+\mathrm{He}, & k_{q 1} \\
\mathrm{CS}_{2}^{*}+\mathrm{CS}_{2} \rightarrow 2 \mathrm{CS}_{2}, & k_{q 2} \\
\mathrm{CS}_{2}^{*}+\mathrm{CS}_{2} \rightarrow 2 \mathrm{CS}+\mathrm{S}_{2}, & k_{r} \\
\mathrm{~S}_{2}+\mathrm{S}_{2} \rightarrow \mathrm{S}_{4}, & k_{2} \\
\mathrm{~S}_{4}+\mathrm{S}_{2} \rightarrow \mathrm{S}_{6} . & k_{3} \\
\mathrm{~S}_{6}+\mathrm{S}_{2} \rightarrow \mathrm{S}_{8}, & k_{4} \\
\mathrm{~S}_{8}+\mathrm{S}_{8} \rightarrow \mathrm{S}_{16}, & k_{2}^{\prime} \\
\mathrm{S}_{16}+\mathrm{S}_{8} \rightarrow \mathrm{S}_{24}, & k_{3}^{\prime} \\
\mathrm{S}_{24}+\mathrm{S}_{8} \rightarrow \mathrm{S}_{32}, & k_{4}^{\prime} \\
" \prime \prime " \text { " " } & " \\
\mathrm{~S}_{8 n-8}+\mathrm{S}_{8} \rightarrow \mathrm{S}_{8 n} & k_{n}^{\prime} \\
\mathrm{S}_{8 n}+p \mathrm{~S}_{8} \rightarrow \mathrm{S}_{8(n+p)}, & \\
\mathrm{S}_{8(n+p)}+q \mathrm{CS}_{2} \rightarrow \text { droplet. } &
\end{array}
$$

The production of $S_{2}$ is still given by Eqs. (1)-(4) above, and the formation of $S_{8}$ is described by Eq. (5). To this point the equation numbers are consistent with those in the text. Equations (A6) and (A7) represent the nucleation and growth of $S_{8}$. Equation (A8) represents the final carbon disulfide growth process to a macroscopic size drop. As indicated by Eq. (9), the production of $S_{2}$ is a slow step in the photoexcitation process. If Eqs. (5a) to $(5 \mathrm{c})$ are irreversible and rapid compared to the production of $S_{2}$ and the nucleation of $S_{8}$ is fast, then the dependence of delay time on excitation intensity will be proportional to the reciprocal of the rate of formation of $S_{8}$. Assuming stationary concentrations 
for the intermediates $S_{2}, S_{4}$, and $S_{6}$ and ignoring diffusive losses (short time behavior) we obtain the following for the rate of formation of $\mathbf{S}_{\mathbf{8}}$ :

$$
d\left(\mathrm{~S}_{8}\right) / d t=\left(k_{r} / 4 k^{\prime}\right)(\sigma I)\left(\mathrm{CS}_{2}\right)^{2} .
$$

Equating the delay time to the reciprocal of Eq. (A9) (using a suitable proportionality constant) and expressing the result in logarithmic form gives an equation of the same form as Eq. (11) in the text.

Now consider the $S_{8}$ nucleation. The kinetic equations for the nucleation step are:

$$
\begin{aligned}
& d\left(\mathbf{S}_{16}\right) / d t=k_{2}^{\prime}\left(\mathbf{S}_{8}\right)\left(\mathbf{S}_{8}\right)-\Omega_{2}^{\prime}\left(\mathbf{S}_{16}\right), \\
& d\left(\mathbf{S}_{24}\right) / d t=k_{3}^{\prime}\left(\mathbf{S}_{16}\right)\left(\mathbf{S}_{8}\right)-\Omega_{3}^{\prime}\left(\mathbf{S}_{24}\right), \\
& d\left(\mathbf{S}_{32}\right) / d t=k_{4}^{\prime}\left(\mathbf{S}_{24}\right)\left(\mathbf{S}_{8}\right)-\Omega_{4}^{\prime}\left(\mathbf{S}_{32}\right), \\
& \left.d\left(\mathbf{S}_{40}\right) / d t=k_{5}^{\prime}\left(S_{32}\right) S_{8}\right)-\Omega_{5}^{\prime}\left(\mathbf{S}_{40}\right), \\
& \quad " \\
& d\left(\mathbf{S}_{8 n-8}\right) / d t=k_{n-1}^{\prime}\left(\mathbf{S}_{8 n-16}\right)\left(\mathbf{S}_{8}\right)-\Omega_{n-1}^{\prime}\left(\mathbf{S}_{8 n-8}\right),
\end{aligned}
$$

(A10m)

$$
d\left(\mathbf{S}_{8 n}\right) / d t=k_{n}^{\prime}\left(\mathbf{S}_{8 n-8}\right)\left(\mathbf{S}_{8}\right),
$$

where $\Omega_{j}^{\prime}=\left[k_{j+1}^{\prime}\left(\mathrm{S}_{8}\right)+k_{D 8 j}\right]$. Equations $(\mathrm{A} 10)$ are identical in structure to the nucleation steps in Eqs. (8) in the text and involve the same assumptions. Following the same procedure used in arriving at Eqs. (12) and (13) in the text, we obtain the following expression for the photoinduced nucleation rate which is identical in structure to Eq. (12)

$$
\begin{aligned}
R & =\frac{k_{n}^{\prime} k_{n-1}^{\prime} \cdots k_{2}^{\prime}\left(V_{c}\right)}{k_{D 8 n-8} k_{D 8 n-16} \cdots k_{D 16}} B^{\prime}\left(S_{8}\right)^{n} \\
& =K_{8}^{\prime}\left(S_{8}\right)^{n} .
\end{aligned}
$$

The steady state concentration of $S_{8}$ is given by

$$
\mathrm{S}_{8}=\frac{k_{r}}{4 k^{\prime} k_{D 8}}(\sigma I)\left(\mathrm{CS}_{2}\right)^{2} .
$$

Substituting Eq. (A12) in Eq. (A11) and expressing the result in logarithmic form gives

$$
\log (R)=n \log (I)+2 n \log \left(\mathrm{CS}_{2}\right)+\log \left(K_{8}\right),
$$

which is identical in form to Eq. (16) in the text.

Clearly, it is possible to identify similar mechanisms for other sulfur allotropes (e.g., $S_{4}$ and $S_{6}$ ) and arrive at similar expressions for the delay time and photoinduced nucleation rate. With the results obtained thus far from our carbon disulfide PIN investigations, we are unable to distinguish between these possibilties.

'D. C. Marvin, H. Reiss, and R. H. Heist, J. Colloid Interface Sci. 58, 125 (1977).

${ }^{2}$ F. C. Wen, T. McLaughlin, and J. L. Katz, Phys. Rev. A 26, 2235 (1982). ${ }^{3}$ J. L. Katz, T. McLaughlin, and F. C. Wen, J. Chem. Phys. 75, 1459 (1981). ${ }^{4}$ J. L. Katz, F. C. Wen, T. McLaughlin, and R. J. Reusch, Science 196, 1203 (1977).

${ }^{5}$ F. C. Wen, T. McLaughlin, and J. L. Katz, Science 200, 769 (1978).

${ }^{6}$ B. Cordier, P. Papan, and J. Lebland, J. Chem. Phys. 74, 3353 (1981).

${ }^{7}$ I. D. Clark and J. F. Noxon, Science 174, 941 (1971).

${ }^{8}$ A. W. Gertler, J. O. Berg, and M. W. El-Sayed, Chem. Phys. Lett. 57, 343 (1978).
${ }^{9}$ A. W. Gertler, B. Almeida, M. A. El-Sayed, and H. Reiss, Chem. Phys. 42, 429 (1979).

${ }^{10}$ H. Reiss, R. H. Heist, R. V. Casberg, and D. C. Marvin (unpublished results); for details contact $R$. $H$. Heist.

${ }^{11}$ R. McGraw and H. Reiss, J. Colloid Interface Sci. 72, 172 (1979).

${ }^{12}$ See, for example, R. H. Heist and H. Reiss, J. Chem. Phys. 59, 665 (1973) or J. L. Katz, J. Chem. Phys. 52, 4733 (1970).

${ }^{13}$ The authors gratefully acknowledge the assistance of Dr. Donald DeClerk of Pfaudler Company, Rochester, N.Y., in the glass coating of the chamber plates.

${ }^{14}$ This coating has been tested at Pfaudler for its resistance to acid attack and found to be roughly 30 times better than Pyrex.

${ }^{15}$ For information concerning the role of gaskets in generating impurities in the TDCC (see Ref. 11).

16. J. Brito, MS Thesis, University of Rochester, 1981.

${ }^{17}$ A. Kacker and R. H. Heist, J. Chem. Phys. 82, 2734 (1985).

${ }^{18}$ The delay would range from 10 's of seconds at the higher carbon disulfide pressures to 100's of seconds at the lower pressures.

${ }^{19}$ W. A. Pryor, Mechanics of Sulfur Reactions (McGraw-Hill, New York, 1962).

${ }^{20}$ Elemental Sulfur, edited by B. Meyer (Interscience, New York, 1965).

${ }^{21}$ Sulfur Research Trends, edited by R. F. Gould (American Chemical Society, Washington, D. C., 1972)

${ }^{22}$ Sulfur in Organic and Inorganic Chemistry, edited by A. Senning (Marcel Dekker, New York, 1972), Vols. 1-3.

${ }^{23}$ Topics in Sulfur Chemistry, edited by A. Senning (Georg Thieme, Stuttgart, 1977), Vols. 1-3.

${ }^{24}$ Inorganic Polymers, edited by F. G. A. Stone and F. Graham (Academic, New York, 1962), Chap. 3.

${ }^{25}$ Chapter 2 in Ref. 19.

${ }^{26}$ Chapter 4 in Ref. 21.

${ }^{27}$ Chapter 5 in Ref. 20.

${ }^{28}$ D. M. Gardner and G. K. Fraenkl, J. Am. Chem. Soc. 78, 3279 (1956).

${ }^{29}$ A. V. Tobolsky and A. Eisenberg, J. Am. Chem. Soc. 81, 780 (1959).

${ }^{30}$ Ibid., 82, 289 (1960).

${ }^{31}$ A. G. Pinkus and L. H. Piette, J. Phys. Chem. 63, 2086 (1959).

${ }^{32}$ Chapter 7 in Ref. 20.

${ }^{33}$ G. Preuner and W. Schupp, Z. Phys. Chem. 68, 129 (1909); H. Braune, S. Peter, and V. Neveling, Z. Naturforsch. Teil A 6, 32 (1951)

${ }^{34}$ P. D. Bartlett and G. Meguerian, J. Am. Chem. Soc. 78, 3710 (1956).

${ }^{35}$ N. R. Dhar and B. V. S. Raghaven, Proc. Nat. Acad. Sci. India Sect. A 17, 7 (1948); Chem. Abstracts 46, 2948d (1952).

${ }^{36}$ P. D. Bartlett, A. K. Calter, R. E. Davis, and W. R. Roderick, J. Am. Chem. Soc. 83, 109 (1961).

${ }^{37}$ Chapter 3 in Ref. 19.

${ }^{38}$ T. E. Ferington and A. V. Tobalsky, J. Am. Chem. Soc. 80, 3215 (1958). ${ }^{39}$ R. J. Kern, J. Am. Chem. Soc. 77, 1382 (1955).

${ }^{40}$ M. S. Kharasch, W. Nudenberg, and T. H. Meltzer, J. Org. Chem. 18, 1233 (1953).

${ }^{41}$ G. Leandri and A. Tundo, Ann. Chim. Rome 44, 63 (1954); Chem. Abstracts 49, 4563d (1955).

${ }^{42}$ T. Otsu, J. Polymer Sci. 21, 559 (1956).

${ }^{43}$ J. Brito and R. H. Heist, Chem. Eng. Commun. 15, 133 (1982).

${ }^{44}$ R. H. Heist, A. Kacker, and J. Brito, Chem. Eng. Commun. 28, 117 (1984).

${ }^{45}$ R. H. Heist and O. Kalisky (unpublished results).

${ }^{46}$ W. A. Noyes and P. A. Leighton, Photochemistry of Gases (Dover, New York, 1941), Chap. IV.

${ }^{47}$ F. J. Wright, J. Phys. Chem. 64, 1648 (1960). See also Ref. 9 and 10 in Wright's paper.

${ }^{48}$ R. J. Richardson, H. T. Powell, and J. D. Kelley, J. Phys. Chem. 77, 2601 (1973).

${ }^{49}$ M. DeSorgo, A. J. Yarwood, O. P. Strausz, and H. E. Gunning, Can. J. Chem. 43, 1886 (1965).

${ }^{50}$ K. Ernst and J. J. Hoffman, Chem. Phys. Lett. 68, 40 (1979).

${ }^{51}$ Chemistry and Physics of Carbon, edited by P. L. Walker (Marcel Dekker, New York, 1965), Vol. 1, pp. 267-283.

${ }^{52}$ L. E. Brus, Chem. Phys. Lett. 12, 116 (1971).

${ }^{53}$ C. Lambert and G. H. Kimbell, Can. J. Chem. 51, 2601 (1973)

${ }^{54}$ J. Heicklen, J. Am. Chem. Soc. 85, 3562 (1963).

${ }^{55}$ G. H. Myers, D. M. Silver, and F. Kaufman, J. Chem. Phys. 44, 718 (1966).

${ }^{56}$ H. Orita, H. Morita, and S. Magakura, Chem. Phys. Lett. 81, 33 (1981).

${ }^{57}$ S. J. Silvers, M. R. McKeever, and G. K. Chawla, in Advances in Laser Chemistry, edited by A. H. Zewail (Springer, New York, 1978), p. 449. 
${ }^{58}$ Typical values used in this calculation are: $\mathrm{CS}_{2}=6.0 \times 10^{18} \mathrm{~cm}^{-3}$; $k^{\prime}=1.3 \times 10^{9} \mathrm{~s} ; \quad \sigma=10^{-19} \mathrm{~cm}^{2} ; \quad I=1.0 \times 10^{14} \mathrm{~cm}^{-2} \mathrm{~s}^{-1} ; k_{r}$ $=6.0 \times 10^{-15} \mathrm{~cm}^{3} \mathrm{~s}^{-1}$. This value of $k_{r}$ was obtained from Ref. 54 above along with data from W. P. Wood and J. Heicklen, J. Phys. Chem. 75, 854 (1971). A second, qualitative estimate of $k_{r}$ is $6.0 \times 10^{-12}$ and is based on observed rates of sulfur production in our cell experiments. Typical (approximate) aerosol particle number densities were 100 particles/cc in about one minute or so with radii of about one micron. Conditions are similar to those cited above. Our estimate of the particle size seems reasonable since the aerosol particles appeared larger than those in a smoke (roughly $0.1 \mu \mathrm{m}$ ) but not quite large enough to settle rapidly (roughly 10 $\mu \mathrm{m})$.

${ }^{59}$ Reference 19, p. 10.
${ }^{60}$ Reference 23, Vol. 2, p. 55.

${ }^{61}$ J. G. Calvert and J. N. Pitts, Photochemistry (Wiley, New York, 1966), pp. 490-491.

${ }^{62}$ Reference 23, Vol. 2, p. 56.

${ }^{63}$ Industrially distilled water was further distilled three more times. First from an alkaline permanganate bath followed by a second distillation. Finally, this water was distilled a third time under helium, and the vapor passed through a quartz tube, which was irradiated with UV light of the same wavelengths used in the experiment, into the TDCC for use in the PIN experiments. Interestingly, when the plain industrially distilled water was used for same experiments with no further purification, the results were the same. 